\title{
PROPIEDADES FÍSICAS Y MECÁNICAS DE COMPUESTOS DE POLIETILENO RECICLADO Y HARINAS DE CORTEZA Y MADERA DE PINUS RADIATA FABRICADOS MEDIANTE MOLDEO POR INYECCIÓN
}

\section{PHYSICAL AND MECHANICAL PROPERTIES OF BARK FLOUR OR WOOD FLOUR OF PINUS RADIATA RECYCLED POLYETHYLENE COMPOSITES}

\author{
Cristian Moya Villablanca ${ }^{1}$, Hernán Poblete Wilson², Luis Valenzuela Hurtado ${ }^{3}$
}

\section{RESUMEN}

Se ensayaron compuestos de polietileno de baja densidad reciclado y harina de madera y de corteza de Pinus radiata $D$. Don, para evaluar el efecto del contenido de harina y la densidad del compuesto sobre la absorción de agua e hinchamiento, dureza Rockwell, resistencia a la tracción y módulo de elasticidad en tracción. Estos compuestos fueron fabricados mediante moldeo por inyección y sin aditivos, en proporciones harina/polietileno de 20/80, 40/60 y 60/40 peso/peso. Se determinaron varias propiedades físicas y mecanicas de los compuestos. Los resultados fueron sometidos a pruebas de comparación de medias y Análisis de Regresión Lineal Múltiple (ARLM), seguido por el método paso a paso o Stepwise (SWM). Los resultados indican que las propiedades físicas aumentaron con el incremento harina, al igual que la rigidez de los compuestos, mientras que la resistencia a la tracción y a la penetración tendieron a disminuir. Los compuestos con harina de corteza fueron más rígidos, absorbieron menos agua y se hincharon menos que los compuestos con harina de madera, en cambio, no se observaron diferencias estadísticas en la dureza, y sólo el compuesto con $20 \%$ de harina de corteza presentó mayor resistencia a la tracción que el resto de los compuestos. El contenido de harina fue la variable que más afecta las propiedades evaluadas, excepto para el E y la dureza de los compuestos con harina de madera, las que fueron mayormente afectadas por su densidad.

Palabras clave: Compuesto, corteza, madera, inyección, sin aditivos, polietileno.

\begin{abstract}
Composites made of recycled low density polyethylene and wood or bark flour of Pinus radiata D. Don were tested, the effect of the flour content and density of the composites on physical and mechanical properties were determinated. These both types of composites were prepared by injection moulding and without chemical additives at 20/80,40/60 and 60/40 of flour/polyethylene (w/w). Several physical and mechanical properties were measured. The results were tested for comparison of means and Multiple Linear Regression Analysis (ARLM), followed by the method stepwise (SWM). The results indicate that the physical properties and the E increased with increasing flour content, while the tensile strength and the hardness tended to decline. The composites made with bark flour had higher stiffness, absorbed less water and swelled less, and show similar hardness than the composite made with wood flour, but only the composite with $20 \%$ (w/w) of bark flour showed greater tensile strength than the others composites. The content of flour was the variable that most affects the properties evaluated, except to the $\mathrm{E}$ and the hardness of the wood flour composites, they were most affected by their density.
\end{abstract}

Keywords: Composite, bark, wood, injection moulding, without additives, polyethylene.

${ }^{1} \mathrm{Ph}$ D. (c) en Ciencias Forestales, Facultad de Ciencias Forestales, Universidad de Concepción. Concepción, Chile

${ }^{2}$ Profesor Titular, Facultad de Ciencias Forestales, Universidad Austral de Chile. Instituto de Tecnología de Productos Forestales, Universidad Austral de Chile, Isla Teja, Valdivia, Chile.

${ }^{3}$ Profesor Asociado, Facultad de Ciencias Forestales, Universidad de Concepción. Facultad de Ciencias Forestales, Laboratorio Silvotecnológico de la madera, Universidad de Concepción, Concepción, Chile.

Autor para correspondencia: cmoya@udec.cl

Recibido: 03. 06. 2011 Aceptado: 16.10. 2011 


\section{INTRODUCCIÓN}

Los materiales compuestos fabricados en base a mezclas de plásticos sintéticos y harinas o fibras vegetales, son de creciente interés en la ciencia e ingeniería de materiales. También, los plásticos reciclados han sido considerados para fabricar nuevas formulaciones de materiales compuestos, debido principalmente a razones ambientales y de costos (English et al. 1997, Wolcott y Englund 1999, Wambua et al. 2003). Algunas de las formulaciones obtenidas pueden presentar similares o mejores propiedades que los mismos plásticos sintéticos, con la ayuda de determinados aditivos químicos, como los agentes acoplantes y plastificantes (Youngquist et al. 1995, Rowell et al. 1998, Jayaraman y Bhattacharyya 2004, Lucchetta et al. 2006, Yeh et al. 2009). Sin embargo, existe escasa información sobre la formulación de compuestos que no contengan estos aditivos químicos. Además, pocas investigaciones se refieren al uso de la corteza como material para fabricar este tipo de compuestos, y de cómo afecta las propiedades del compuesto resultante.

La presente investigación tuvo como objetivo determinar las propiedades físicas: densidad, contenido de humedad, absorción de agua e hinchamiento a 2 y $24 \mathrm{~h} \mathrm{a} 25^{\circ} \mathrm{C}$; y las propiedades mecánicas: resistencia a la tracción, módulo de elasticidad en tracción y dureza Rockwell, de los compuestos de polietileno de baja densidad reciclado (PEr) y harina de corteza y de madera de Pinus radiata D. Don, fabricados en diferentes proporciones (peso/peso), mediante moldeo por inyección y sin ningún tipo de aditivos químicos. Además, se determinó el efecto del tipo y contenido de harina y la densidad del compuesto sobre las propiedades físicas y mecánicas evaluadas.

\section{METODOLOGÍA}

\section{Materiales}

Polietileno. La matriz termoplástica corresponde a polietileno de baja densidad reciclado (PEr), obtenido en el mercado en forma de pellets de $6.5 \mathrm{~mm}$ de largo y $2 \mathrm{~mm}$ de diámetro, y con una densidad de $0.94 \mathrm{~g} / \mathrm{cm}^{3}$, y un índice de fluidez (MFI) de $20 \mathrm{~g} / 10 \mathrm{~min}$, según extrusora con salida de $2 \mathrm{~mm}$ de diámetro y $8 \mathrm{~mm}$ de largo y carga de $2.16 \mathrm{~kg}$, previo calentamiento a $190^{\circ} \mathrm{C}$, durante $5 \mathrm{~min}$.

Madera. La corteza y madera se obtuvieron a partir de desechos del aserrío del primer trozo de un árbol de 22 años de la especie Pinus radiata D. Don cosechado en la zona de Constitución, Región del Maule, Chile. La diferencia química que existe entre la corteza y la madera está claramente definida para esta especie, (Tabla 1). La corteza presenta mayor contenido de extraíbles, menor contenido de celulosa cristalina y de lignina condensada y mayor contenido de hemicelulosa y de fenoles de menor masa molecular que los presentes en la madera.

Tabla 1. Composición química de madera y corteza de Pinus radiata D. Don (Hakki y Kelley 2002, Berrocal et al. 2004).

\begin{tabular}{lcc}
\hline \multicolumn{1}{c}{ Componentes } & Corteza $\left(\% \mathbf{b p s}^{\mathbf{x}}\right)$ & Madera $^{\left(\% \mathbf{b p s}^{\mathbf{x}}\right)}$ \\
\hline Carbohidratos no celulósicos & $12-18^{1 *}$ & $15-20^{1}$ \\
Celulosa & $30-40^{*}$ & $40-50$ \\
Lignina Soluble & $0.1-0.8$ & $0.2-0.5$ \\
Lignina Klason & $20-35$ & $22-40$ \\
Extraíbles & $5-12^{2}$ & $0-4^{3}$ \\
\hline
\end{tabular}

${ }^{1}$ glucomananos; ${ }^{2}$ Principalmente taninos condensados; ${ }^{3}$ Principalmente extraíbles solubles en solventes orgánicos; ${ }^{*}$ compuestos de baja masa molecular. ${ }^{\times}$base peso seco. 
Equipos. Estación de inyección Po Yuen E 400 con fuerza de cierre de 400 tm y capacidad de inyección máxima de 1.500 g Máquina Universal de ensayos Metrotec, con una celda de carga de 10 kgf. Molino de tambor de 4 cuchillos y de $975 \mathrm{rpm}$ marca Wittenheim. Molino de 6 ejes y 12 martillos por eje, y de $3500 \mathrm{rpm}$ marca Wittenheim. Secador de aire caliente por resistencia eléctrica de 100 L marca Po Yuen.

\section{MÉTODO}

Preparación de harina. La corteza y los desechos de madera fueron molidos mediante molinos de cuchillos y de martillos con un 10-12\% de contenido de humedad inicial (CHi), para obtener tamaños de partícula menores a $0.5 \mathrm{~mm}$ (US Standard mesh № 35). Los tamaños de las harinas obtenidos fueron: harina de corteza $=43-52 \mu \mathrm{m}$ de largo y $24-29 \mu \mathrm{m}$ de ancho; harina de madera $=36-43 \mu \mathrm{m}$ de largo y 11-14 $\mu \mathrm{m}$ de ancho. Estos tamaños son mucho menores a la mayoría de las harinas utilizadas comercialmente para fabricar este tipo de compuestos, las que fluctúan normalmente entre 50 y 800 $\mu \mathrm{m}$ de largo (Stark 1999, Bouafif et al. 2009a, Ngueho et al. 2010), y son similares a los tamaños de los talcos utilizados normalmente como material de relleno (Sanadi et al. 1999). Posteriormente a la molienda, ambas harinas fueron secadas hasta un $3 \%(\mathrm{p} / \mathrm{p})$ de $\mathrm{CH}$ y mantenidas con esa humedad hasta ser procesadas.

Obtención del compuesto. Un total de 7 formulaciones fueron fabricadas mediante moldeo por inyección y sin agentes acoplantes u otro tipo de aditivos. Tres formulaciones de PEr con 20, 40 y $60 \%$ peso/peso (p/p) de harina de madera (M20, M40 y M60, respectivamente). Tres formulaciones de PEr con 20, 40 y 60\% (p/p) de harina de corteza (C20, C40 y C60, respectivamente). Además de la muestra testigo fabricada $100 \%$ de PEr. Un total de diez muestras para cada formulación fueron procesados con las harinas a un $3 \%(\mathrm{p} / \mathrm{p})$ de $\mathrm{CH}$, utilizando una temperatura de tornillo de inyección a $190^{\circ} \mathrm{C}$ en las seis estaciones y una presión máxima de moldeo de $120 \mathrm{~kg} / \mathrm{cm}^{2}$. Todas las probetas presentaron la misma orientación de las harinas dentro de la matriz, debido a que los contenedores utilizados como muestras fueron obtenidos bajo las mismas condiciones de procesamiento, además, las probetas para la determinación de las propiedades fueron obtenidas de la misma zona de cada contenedor, para anular alguna posible variabilidad de la mezcla generada durante el proceso de fabricación (Kim et al. 2008).

\section{Determinación de las propiedades de los compuestos}

El contenido de humedad $(\mathrm{CH})$, la densidad de referencia o aparente $(\rho)$, la absorción e hinchamiento en agua y la dureza Rockwell de cada compuesto, incluyendo las muestras testigos de PEr, fueron determinados utilizando 3 probetas obtenidas de 10 contenedores elegidos en forma aleatoria. Para la determinación de la resistencia a la tracción y el módulo de elasticidad en tracción (E) se utilizaron 5 probetas elegidas de la misma forma. Todas las probetas fueron climatizadas a $20 \pm 3^{\circ} \mathrm{C}$, con $60 \pm 5 \%$ de humedad relativa, para ser evaluadas según las normas respectivas, excepto para calcular el $\mathrm{CH}$, el que fue determinado inmediatamente después de fabricar cada compuesto.

\section{Propiedades físicas y mecánicas.}

Las propiedades medidas fueron:

* Contenido de humedad: De acuerdo con NCh 761

* Densidad aparente: De acuerdo con ASTM D 792-08

* Absorción e hinchamiento a 24 h: Inmersión en agua a 25 y $90{ }^{\circ} \mathrm{C}$ según ASTM 570-98(2010e1)

* Resistencia a la tracción y módulo de elasticidad en tracción (E): Según norma ASTM D 638-10

* Dureza: De acuerdo con ASTM D 785-08 para espesor de $3 \mathrm{~mm}$. 


\section{Análisis Estadístico}

Los resultados obtenidos fueron evaluados utilizando el programa Statistica 6.0, determinando los valores medios de cada propiedad y sus diferencias, mediante pruebas estadísticas de mínima diferencia significativa (LSD) de Fisher. Además, se determinaron los efectos del contenido de harina y de la densidad de los compuestos sobre la absorción e hinchamiento, resistencia a la penetración y a la tracción y el E de los compuestos, mediante Análisis de Regresión Lineal Múltiple (ARLM) utilizando el método stepwise (SWM) (Valenzuela et al. 1999).

\section{RESULTADOS}

\section{Propiedades físicas de los compuestos}

\section{Contenido de Humedad (CH)}

En la tabla 2, columna 2, se presentan los valores promedios y la desviación estándar del $\mathrm{CH}$ de los compuestos. En esta tabla se puede observar que el $\mathrm{CH}$ de los compuestos es mayor al del termoplástico testigo (PEr), y que a medida que el contenido de harina se incrementó, el CH aumentó hasta estabilizarse entre los compuestos con 40 y $60 \%(\mathrm{p} / \mathrm{p})$ de harina. Es importante notar que el $\mathrm{CH}$ inicial de todas las harinas antes del procesamiento no fue superior al $3 \%$, y que todos los compuestos aumentaron el $\mathrm{CH}$ después de su fabricación. Para los compuestos con harina de madera, este incremento del $\mathrm{CH}$ varió desde 3.47 a $6.44 \%$, para compuestos con $20 \%$ y $40 \%$ (p/p) de harina, respectivamente, estabilizando su $\mathrm{CH}$ a $6.78 \%$ para compuestos con $60 \%(\mathrm{p} / \mathrm{p})$ de harina. Para los compuestos con harina de corteza, el CH aumentó de 2.63 a $6.55 \%$, para compuestos con $20 \%$ y $40 \%$ (p/p) de harina, respectivamente, estabilizando su $\mathrm{CH}$ a $6.82 \%$ para los compuestos con $60 \%(\mathrm{p} / \mathrm{p})$ de harina. Contenidos de humedades similares han sido reportado por varios autores, para compuestos fabricados en base a harinas de madera y termoplásticos mediante moldeo por inyección (Rowell et al. 2000, Raukola y Makinen 2003, Dominkovics et al. 2007). Este aumento del CH en el compuesto después de su fabricación puede deberse a dos factores; a) a la naturaleza higroscópica de estas harinas, debido a la presencia de los grupos hidroxilos $(\mathrm{OH})$ de los carbohidratos que forman la pared celular de las fibras vegetales, tales como celulosa y hemicelulosa (Sanadi et al. 1995, Rowell et al. 2005, Beg y Pickering 2008a y 2008b) a la baja calidad de encapsulamiento de las partículas por parte del termoplástico producida durante la fabricación del compuesto, la que deja a las partículas de harinas absorban la humedad del medio ambiente hasta llegar a la humedad de equilibrio (Wolcott y Englund 1999, Yadama et al. 2009). En este caso, el CH de equilibrio de las harinas varió entre 10 y $15 \%(\mathrm{p} / \mathrm{p})$ para contenidos de 60 y $20 \%$ (p/p), respectivamente, si se considera que el agua del compuesto está presente sólo en las harinas.

En tabla 2, columna 2, también se puede observar, que no existe diferencia estadística del $\mathrm{CH}$ entre los compuestos fabricados con diferente tipo de harina y a iguales proporciones. La literatura indica que en las especies del género Pinus, la corteza generalmente presenta un $\mathrm{CH}$ menor que la madera, debido a su mayor contenido de extraíbles hidrofóbicos (Hon y Shiraishi 2000, Rowell et al. 2005). Aunque, Poblete y Sánchez (1991), reportaron un mayor CH en partículas de corteza que en partículas de madera, determinados antes de fabricar los tableros de partículas de la especie Pinus radiata $\mathrm{D}$. Don, y después de secar al $3 \%$. En este caso, el alto $\mathrm{CH}$ observado en los compuestos fabricados con harina de corteza, puede ser atribuido a que las altas temperaturas a que fueron sometidas las harinas durante la molienda, secado y procesamiento $\left(160\right.$ a $\left.190^{\circ} \mathrm{C}\right)$, pueden degradar los extraíbles y carbohidratos de menor masa molecular presentes en mayor cantidad que en la madera (Beall y Eickner 1970, Sjöström 1993, Hon y Shiraishi 2000). Los gases y el vapor de agua resultante puede encapsularse por la matriz termoplástica, durante el proceso de moldeo (Guo et al. 2004, Shebani et al. 2009, Bouafif et al. 2009a), favoreciendo el aumento del CH de los compuestos. 
Tabla 2. Propiedades físicas de los compuestos.

\begin{tabular}{|c|c|c|c|c|c|c|}
\hline \multirow[b]{2}{*}{ Formulación } & \multirow[b]{2}{*}{$\mathrm{CH}(\%)$} & \multirow[b]{2}{*}{$\rho\left(\mathrm{g} / \mathrm{cm}^{3}\right)$} & \multicolumn{2}{|c|}{ Absorción de água (\%) } & \multicolumn{2}{|c|}{ Hinchamiento (\%) } \\
\hline & & & $25^{\circ} \mathrm{C}$ & $90^{\circ} \mathrm{C}$ & $25^{\circ} \mathrm{C}$ & $90^{\circ} \mathrm{C}$ \\
\hline PEr & $\begin{array}{l}\mathbf{0 . 0 8} \text { a } \\
(0.14)\end{array}$ & $\begin{array}{l}\mathbf{0 . 9 4 1} \mathbf{a} \\
(0.003)\end{array}$ & $\begin{array}{l}3^{\star} \mathbf{1 0}^{-3} \mathbf{a} \\
(0.0006)\end{array}$ & $\begin{array}{l}12^{\star} 10^{-3} \mathbf{a} \\
(0.0026)\end{array}$ & $\begin{array}{l}34^{\star} 10^{-3} \mathbf{a} \\
(0.0035)\end{array}$ & $\begin{array}{c}\mathbf{1 2 8}^{\star} \mathbf{1 0}^{-3} \mathbf{a} \\
(0.0056)\end{array}$ \\
\hline 20\%Madera & $\begin{array}{l}3.47 \text { b } \\
(0.54)\end{array}$ & $\begin{array}{l}\mathbf{0 . 9 8 8} \mathbf{a} \\
(0.010)\end{array}$ & $\begin{array}{c}\mathbf{2 3}^{\star} \mathbf{1 0 ^ { - 3 }} \mathbf{a b} \\
(0.0006)\end{array}$ & $\begin{array}{c}220^{\star} \mathbf{1 0}^{-3} \mathbf{a b} \\
(0.0038)\end{array}$ & $\begin{array}{l}\mathbf{7 7}^{\star} 10^{-3} \mathbf{a} \\
(0.0035)\end{array}$ & $\begin{array}{c}\mathbf{2 7 3}^{\star} \mathbf{1 0}^{-3} \mathbf{a} \\
(0.0114)\end{array}$ \\
\hline 40\%Madera & $\begin{array}{l}6.44 \mathrm{c} \\
(0.48)\end{array}$ & $\begin{array}{c}\mathbf{1 . 0 4 9} \mathbf{b} \\
(0.015)\end{array}$ & $\begin{array}{c}150^{\star} 10^{-3} \mathbf{c} \\
(0.0046)\end{array}$ & $\begin{array}{c}\mathbf{6 6 0}^{\star} \mathbf{1 0}^{-3} \mathbf{c} \\
(0.0171)\end{array}$ & $\begin{array}{c}\mathbf{1 8 2}^{\star} \mathbf{1 0}^{-3} \mathbf{b} \\
(0.0056)\end{array}$ & $\begin{array}{c}\mathbf{6 6 7}^{\star} \mathbf{1 0}^{-\mathbf{3}} \mathbf{b} \\
(0.0199)\end{array}$ \\
\hline $60 \%$ Madera & $\begin{array}{l}6.78 \mathrm{c} \\
(1.40)\end{array}$ & $\begin{array}{l}\mathbf{1 . 0 4 9} \mathbf{b} \\
(0.059)\end{array}$ & $\begin{array}{c}\mathbf{4 1 3}^{\star} \mathbf{1 0}^{-\mathbf{3}} \mathbf{d} \\
(0.0117)\end{array}$ & $\begin{array}{c}1140^{*} \mathbf{1 0}^{-3} \mathbf{d} \\
(0.0104)\end{array}$ & $\begin{array}{c}\mathbf{2 9 9}^{\star} \mathbf{1 0}^{-3} \mathbf{c} \\
(0.0011)\end{array}$ & $\begin{array}{c}\mathbf{1 0 8 1}^{\star} \mathbf{1 0}^{-\mathbf{3}} \mathbf{c} \\
(0.0073)\end{array}$ \\
\hline $20 \%$ Corteza & $\begin{array}{l}2.63 \mathbf{b} \\
(0.24)\end{array}$ & $\begin{array}{l}\mathbf{0 . 9 7 4} \mathbf{a} \\
(0.008)\end{array}$ & $\begin{array}{c}\mathbf{2 0} \mathbf{1 0}^{-3} \mathbf{a b} \\
(0.0010)\end{array}$ & $\begin{array}{c}\mathbf{1 7 0}^{*} \mathbf{1 0}^{-3} \mathbf{a b} \\
(0.0006)\end{array}$ & $\begin{array}{l}\mathbf{5 7}^{\star} \mathbf{1 0 ^ { - 3 }} \mathbf{a} \\
(0.0015)\end{array}$ & $\begin{array}{c}\mathbf{2 0 9}^{\star} \mathbf{1 0}^{-3} \mathbf{a} \\
(0.0046)\end{array}$ \\
\hline $40 \%$ Corteza & $\begin{array}{l}6.55 \mathrm{c} \\
(2.03)\end{array}$ & $\begin{array}{l}\mathbf{1 . 0 4 8} \mathbf{b} \\
(0.014)\end{array}$ & $\begin{array}{c}\mathbf{7 7 ^ { \star }} \mathbf{1 0}^{-\mathbf{3}} \mathbf{a b c} \\
(0.0037)\end{array}$ & $\begin{array}{c}\mathbf{3 2 0}^{\star} \mathbf{1 0}^{-3} \mathbf{b} \\
(0.0015)\end{array}$ & $\begin{array}{l}\mathbf{6} \mathbf{1}^{\star} \mathbf{1 0 ^ { - 3 }} \mathbf{a} \\
(0.0044)\end{array}$ & $\begin{array}{c}\mathbf{3 0 0}^{\star} \mathbf{1 0}^{-3} \mathbf{a} \\
(0.0041)\end{array}$ \\
\hline $60 \%$ Corteza & $\begin{array}{l}6.82 \mathrm{c} \\
(0.28)\end{array}$ & $\begin{array}{l}1.121 \mathrm{c} \\
(0.030)\end{array}$ & $\begin{array}{c}\mathbf{9 3}^{\star} \mathbf{1 0}^{-3} \mathbf{b c} \\
(0.0015)\end{array}$ & $\begin{array}{c}\mathbf{7 7 0}^{\star} \mathbf{1 0}^{-3} \mathbf{c} \\
(0.0180)\end{array}$ & $\begin{array}{c}\mathbf{9 7}^{\star} \mathbf{1 0}^{-3} \mathbf{a b} \\
(0.0031)\end{array}$ & $\begin{array}{c}\mathbf{7 1 7}^{\star} \mathbf{1 0}^{-\mathbf{3}} \mathbf{b} \\
(0.0121)\end{array}$ \\
\hline
\end{tabular}

Letras diferentes en cada columna indican diferencias significativas, Fisher $\alpha=0.05$.

Número entre paréntesis $($ ) = Desviación estándar.

\section{Densidad aparente o de referencia de los compuestos $(\rho)$}

Los valores promedios de la $\rho$ del PEr y de los compuestos evaluados se presentan en tabla 2, columna 3. En esta tabla se observa que la densidad aumentó a medida que se incrementó el contenido de ambos tipos de harinas, comparado con la densidad del PEr $\left(0.940 \mathrm{~g} / \mathrm{cm}^{3}\right)$, variando desde 0.988 a $1.049 \mathrm{~g} / \mathrm{cm}^{3}$, para los compuestos en base a madera y de 0.974 a $1.121 \mathrm{~g} / \mathrm{cm}^{3}$, para los compuestos en base a corteza. Valores similares han sido reportados por otros investigadores, para compuestos de termoplásticos y harinas de madera de diferentes especies, fabricados mediante moldeo por inyección, extrusión y compresión (Stark 1997, English et al. 1997, Stark 1999, Rowell et al. 1998, Beg y Pickering 2008b, Kuo et al. 2009, Ngueho et al. 2010). Este aumento de densidad fue atribuido principalmente al colapso de los lúmenes y espacios vacíos de las partículas de harinas, que ocurre normalmente durante el proceso de fabricación (Matuana et al. 1997, Stark 1997), junto con la mayor densidad de la pared celular de las harinas, comparado con la densidad del PEr. Además, este aumento de densidad es mayor en los compuestos con mayor contenido de harina, debido a la mayor cantidad de espacios vacíos disponibles para ser colapsados durante el proceso de fabricación (English et al. 1997, Raukola y Makinen 2003, Meran et al. 2008).

En tabla 2, columna 3, también se puede observar que los compuestos con $60 \%(\mathrm{p} / \mathrm{p})$ de harina de corteza son más densos que los compuestos con $60 \%(\mathrm{p} / \mathrm{p})$ de harina de madera. Esta diferencia puede ser atribuida a que ambos tipos de partículas de harina son químicamente distintas, y por lo tanto, responden de forma diferente a la compresión y al colapso (Rizvi et al. 2000). Sin embargo, Ngueho et al. (2010) reportaron un comportamiento contrario en compuestos con similares contenidos de harina de Pinus ssp en una matriz de polipropileno, donde los compuestos en base a madera son más 
densos que los compuestos fabricados con harina de corteza, tanto de coníferas como de latifoliadas. Esta diferencia fue atribuida a la mayor cantidad de celulosa cristalina y lignina condensada presentes en la madera, comparado con las existentes en la corteza. Aunque también, hay que considerar que la humedad presente en las harinas (3\%) utilizadas en esta investigación, fueron sometidas a altas temperaturas durante el moldeo, pudiendo promover la formación de burbujas durante el procesamiento, dejando espacios vacíos en la matriz y/o en la interface fibra-matriz, los que pueden alterar la densidad del compuesto (Beall y Eickner 1970, Stark 1999, Rizvi et al. 2000).

\section{Absorción de agua e hinchamiento de los compuestos}

Los resultados de la absorción e hinchamiento de los compuestos sometidos a inmersión en agua durante $24 \mathrm{~h}$, a $25^{\circ} \mathrm{C}$, se presentan en las columnas 4 y 6 de la tabla 2. En esta tabla se puede observar que la absorción e hinchamiento de los compuestos fueron mayores que los observados en el PEr, siendo este casi nulo $(0.003 \% \mathrm{p} / \mathrm{p})$. También, se puede observar que estas propiedades aumentaron con el incremento del contenido de harina, tanto de madera como de corteza. El aumento de la absorción varió desde $0.023 \% \mathrm{p} / \mathrm{p}$ a $1.14 \% \mathrm{p} / \mathrm{p}$, para los compuestos en base a harina de madera, $\mathrm{y}$ desde $0.020 \% \mathrm{p} / \mathrm{p}$ a $0.77 \% \mathrm{p} / \mathrm{p}$ para los compuestos en base a harina de corteza. Para el caso del hinchamiento, este varió desde $0.077 \% \mathrm{p} / \mathrm{p}$ a $1.08 \% \mathrm{p} / \mathrm{p}$, para los compuestos en base a harina de madera, y desde $0.0057 \%$ p/p a $0.717 \%$ p/p, para los compuestos en base a harina de corteza. Estos resultados son menores a los reportados por varios investigadores en compuestos fabricados por inyección, extrusión y compresión, con similares contenidos de harinas y diferentes termoplásticos, y sometidos a las mismas condiciones experimentales (Wang y Morrell 2004, Rowell et al. 2005, Yang et al. 2006a, Zabihzadeh 2010, Ashori y Nourbakhsh 2010).

En tabla 2, también se puede observar diferencias estadísticas de absorción e hinchamiento entre los compuestos fabricados con iguales contenidos de harina. Los compuestos que contienen harina de corteza presentan menores valores que los compuestos fabricados con harina de madera, excepto para los compuestos con $20 \%$ p/p de harina, los que no presentan diferencias estadísticas en ambas propiedades. Resultados similares han sido reportados por Najafi et al. (2008) y Bouafif et al. (2009a), quienes encontraron que los compuestos de PE de alta y baja densidad, polipropileno y harinas de madera de coníferas y latifoliadas, fabricados con y sin agentes acoplantes mediante moldeo por extrusión e inyección, también presentan mayor absorción de agua que los compuestos fabricados en base a harina de corteza. Estas diferencias podrían estar relacionada a dos factores; a) a la mayor capacidad higroscópica de la madera, generada por la mayor cantidad de grupos $\mathrm{OH}$ presentes en la superficie de sus paredes celulares, en comparación con los disponibles en la harina de corteza. Además, la corteza contiene mayor cantidad de extraíbles del tipo hidrofóbico, que pudiera repeler el agua, aunque gran parte de estos pudieron ser evaporados durante el proceso de fabricación del compuesto (Neagu et al. 2005, Demir et al. 2006, Rahman et al. 2009) a la presencia de espacios vacíos de los lúmenes no colapsados y de las zonas de fallas, normalmente producidas entre la harina y la matriz durante la fabricación del compuesto, los que pueden influenciar los procesos de difusión del agua en los compuestos (Yang et al. 2006a, Panthapulakkal y Sain 2007).

Para el caso de los ensayos de absorción e hinchamiento a $90^{\circ} \mathrm{C}$, los resultados se presentan en tabla 2, columna 5 y 7 . En esta tabla se observa una mayor absorción y un mayor hinchamiento en los compuestos evaluados a $90^{\circ} \mathrm{C}$ que a $25^{\circ} \mathrm{C}$ (ver columna 4 y 6), para todos los compuestos, manteniéndose una tendencia similar a la observada en los ensayos efectuado a $25^{\circ} \mathrm{C}$. Los compuestos fabricados con harina de corteza tienden a absorber menos agua e hincharse menos que los compuestos fabricados con harina de madera, pero los valores son muy superiores a los obtenidos a $25^{\circ} \mathrm{C}$, hasta 4 veces lo absorbido e hinchado. Este comportamiento se debe a que la temperatura aumenta la capacidad de difusión del agua en las harinas, así como también, la dilatación del PEr (Kandem et al. 2004, Akil et al. 2009). Estos resultados son similares a los obtenidos en compuestos fabricados por 
inyección, y son mucho menores a los compuestos fabricados por extrusión y compresión (Rowell et al. 1998, Rahman et al. 2009, Kuo et al. 2009, Zabihzadeh 2010).

\section{Propiedades mecánicas de los compuestos}

\section{Resistencia a la tracción}

En tabla 3, columna 3, se presentan los resultados de la resistencia a la tracción de ambos tipos de compuestos. Se observa que la resistencia a la tracción de los compuestos disminuye con el incremento del contenido de ambos tipos harinas. Bledzki y Faruk (2004), Yang et al. (2006)a, Ngueho et al. (2010) y Zabihzadeh (2010), reportaron similar comportamiento en compuestos con harinas de maderas y sin agentes acoplantes, el que fue atribuido a la disminución de la matriz plástica debido al aumento del contenido de harina en el compuesto, y a la pobre adhesión entre la matriz y las harinas, las que disminuyen aún más con el incremento de harina en el compuesto. Sin embargo, los valores de resistencia a la tracción de los compuestos obtenidos en el presente estudio (17 a 41 $\mathrm{MPa}$ ), son muy superiores a los indicados como aceptables por US Plastic Lumber para compuestos fabricados por extrusión con $65 \%$ de fibras de madera y termoplásticos reciclados $5 \mathrm{MPa}$ (US Plastic Lumber, 2004) y son similares a la mayoría de los compuestos fabricados por inyección, extrusión y compresión, con diferentes termoplásticos, vírgenes y reciclados, con y sin agentes acoplantes (English 1999, Stark 1999, Clemons 2002, Selke y Wishman 2004, Kuan et al. 2006, Yang et al. 2006b, Beg y Pickering 2008a, Kuo et al. 2009, Yeh et al. 2009).

Tabla 3. Propiedades mecánicas de los compuestos.

\begin{tabular}{|c|c|c|c|c|}
\hline Compuesto & $\rho\left(\mathrm{g} / \mathrm{cm}^{3}\right)$ & $\begin{array}{l}\text { Resistencia a la tracción } \\
\qquad(\mathrm{MPa})\end{array}$ & $\begin{array}{c}E \\
(\mathrm{GPa})\end{array}$ & Dureza (kgf) \\
\hline \multirow{2}{*}{ PEr } & 0.941 a & $40.925 a$ & $6.20 \mathrm{a}$ & 164.496 a \\
\hline & $(0.003)$ & $(1.468)$ & $(0.41)$ & $(25.221)$ \\
\hline \multirow{2}{*}{ 20\%Madera } & $0.988 a$ & $30.174 \mathrm{~b}$ & $6.85 \mathrm{ab}$ & 160.044 a \\
\hline & $(0.010)$ & $(2.747)$ & $(0.66)$ & $(26.526)$ \\
\hline \multirow{2}{*}{ 40\%Madera } & $1.049 \mathrm{~b}$ & $26.733 \mathrm{~b}$ & $7.93 \mathrm{bc}$ & $146.132 \mathrm{ab}$ \\
\hline & $(0.015)$ & $(2.637)$ & $(0.21)$ & (19.375) \\
\hline \multirow{2}{*}{$60 \%$ Madera } & $1.049 \mathrm{~b}$ & $20.683 c$ & $8.87 \mathrm{c}$ & $123.516 \mathrm{~b}$ \\
\hline & $(0.059)$ & $(2.990)$ & $(0.64)$ & $(21.692)$ \\
\hline \multirow{2}{*}{ 20\%Corteza } & $0.974 \mathrm{a}$ & $38.674 \mathrm{a}$ & $9.13 \mathrm{c}$ & $145.304 \mathrm{ab}$ \\
\hline & $(0.008)$ & $(0.246)$ & $(0.64)$ & $(20.929)$ \\
\hline \multirow{2}{*}{$40 \%$ Corteza } & $1.048 \mathrm{~b}$ & $26.725 \mathrm{~b}$ & $9.16 \mathrm{c}$ & $155.200 \mathrm{a}$ \\
\hline & $(0.014)$ & $(2.229)$ & $(0.34)$ & $(23.440)$ \\
\hline \multirow{2}{*}{$60 \%$ Corteza } & $1.121 \mathrm{c}$ & $17.235 \mathrm{c}$ & $9.20 \mathrm{c}$ & $123.314 \mathrm{~b}$ \\
\hline & $(0.030)$ & (4.128) & $(0.59)$ & $(28.537)$ \\
\hline
\end{tabular}

* Letras diferentes dentro de cada columna indican diferencias significativas, Fisher $\alpha=0.05$ Número entre paréntesis ( ) = Desviación estándar. 
Es posible observar (Tabla 3) que no existen diferencias estadísticas entre los compuestos fabricados tanto con corteza como con madera, a iguales contenidos de harinas. Sin embargo, una excepción interesante se observa en los compuestos que contienen $20 \%(\mathrm{p} / \mathrm{p})$ de harina de corteza. Estos no presentan diferencias estadísticas de resistencia con el PEr y son muy superiores a los otros compuestos, ver tabla 3, columna 3, fila 2 a 6 . Esta diferencia puede estar relacionada con la condensación producida por taninos u otros compuestos fenólicos que se encuentran mayormente presentes en la corteza (Hakki y Kelley 2002, Nemli et al. 2004, Rowell et al. 2005). Aunque, el efecto de la condensación no se ve reflejado en los compuestos con mayor contenido de harina. Esto puede ser explicado por la inadecuada dispersión de la harina dentro de la matriz que se produce en los compuestos con altos contenidos de harina, generando zonas de fallas que pueden afectar las propiedades mecánicas del compuesto (Renner et al. 2009, Ngueho et al. 2010).

\section{Módulo de elasticidad en tracción (E)}

En tabla 3, columna 4, se presentan los resultados del módulo de elasticidad en tracción (E) de ambos tipos de compuestos. En esta tabla se observa que todos los compuestos presentan mayor E que el PEr, sin embargo, sólo los compuestos en base a harina de madera presentan un aumento del E proporcional al incremento de harina, mientras que los compuestos con corteza no presentan diferencias estadísticas según su contenido de harina. Resultados similares han sido reportados por varios autores en investigaciones de compuestos con diversas matrices termoplásticas y harinas o fibras de maderas y cortezas, con y sin agentes acoplantes (Woodhams et al. 1984, Zadorecki y Mitchell 1989, Bozaci et al. 2007, Ngueho et al. 2010). Además, los compuestos fabricados con harina de corteza presentan mayor E que los compuestos fabricados con harina de madera. Ngueho et al. (2010) reportaron resultados contrarios en compuestos plástico y fibras de corteza de conífera y latifoliada, fabricados con agentes acoplantes y con alto contenido de talco, este último tipo de aditivo tiende a aumentar aún más el módulo de elasticidad de los compuestos (Lee et al. 2008).

En figura 1a y 1b, se grafican las curvas promedio de fuerza-extensión (elongación) de los compuestos fabricados con ambos tipos de harinas. En estos gráficos, se puede observar que la carga máxima y la extensión máxima disminuyen al aumentar el contenido de harina en ambos tipos de compuestos. Por otro lado, en la figura 1a, se puede observar que las pendientes en la zona elástica de las curvas esfuerzo-deformación aumentan gradualmente al aumentar el contenido de harina de madera, esto significa que un mayor contenido de harina de madera aumenta la rigidez del compuesto (Tabla 3, columna 4, filas 3 a 5). Sin embargo, este comportamiento es diferente para los compuestos fabricados con harina de corteza (Figura 1b), donde la rigidez de estos compuestos aumenta y se mantiene constante con el aumento del contenido de harina (Tabla 3, columna 4, filas 6 a 8).
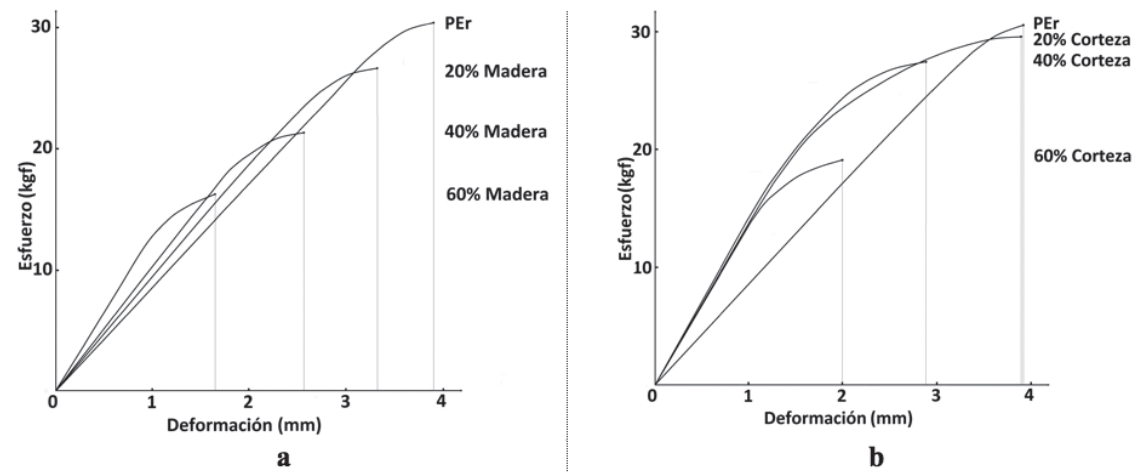

Figura 1. Curvas promedio de esfuerzo-deformación de los compuestos: a) en base a harina de madera, y b) en base a harina de corteza. 
De acuerdo a los resultados presentados en tabla 3, columna 4, los compuestos con $20 \%$ de harina de corteza presentaron mayor rigidez que los compuestos fabricados con harina de madera y que la presentada por el PEr, ver filas 2 y 6 y similar rigidez que los compuestos fabricados con 40 y $60 \%$ de harina de corteza (ver filas 7 y 8). Al igual que la mayor resistencia a la tracción de los compuestos con $20 \%$ de harina de corteza, la mayor rigidez de estos compuestos puede estar relacionada con la condensación de los taninos u otros compuestos fenólicos que se encuentran mayormente presentes en la corteza (Hakki y Kelly 2002, Nemli et al. 2004, Rowell et al. 2005), junto con la inadecuada dispersión de la harina dentro de la matriz de los compuestos con altos contenidos de harina (Renner et al. 2009, Ngueho et al. 2010). Aunque también, esta diferencia podría ser atribuida a la desigual estructura química de las superficies de las paredes celulares de la corteza y de la madera, algunas investigaciones reportan que esta diferencia química podría afectar la cristalización de la matriz termoplástica (material semicristalino), en consecuencia, la rigidez del compuesto (Harper y Wolcott 2004, Rowell et al. 2005, Demir et al. 2006, Bouafif et al. 2009b, Ngueho et al. 2010). Sin embargo, otros autores indican que la presencia de los grupos $\mathrm{OH}$ en la madera y en cualquier otro tipo de tejido vegetal, no compatibilizan químicamente con los grupos hidrofóbicos presentes en las polioleofinas, como en los termoplásticos (Yin et al. 2001, Guo et al. 2004, Gardner et al. 2008, Godara et al. 2009). La discusión anterior requiere la comprobación de la existencia de una diferente formación de la microestructura en la interfase harina-matriz termoplástica, según el tipo de harina y la historia térmica del compuesto.

\section{Dureza}

En tabla 3, columna 4, se presentan los resultados de dureza Rockwell de los compuestos. De acuerdo con estos resultados, la dureza tiende a disminuir con el incremento de harina, para ambos tipos de harinas. Sin embargo, sólo los compuestos con mayor contenido de harina (60\%), tanto de corteza como de madera, presentan valores de dureza estadísticamente menores que el PEr. Sin embargo, otros estudios indican un aumento de la dureza del compuesto con el incremento de harina o fibras (Rowell et al. 1998, Wang et al. 2003). Además, en esta tabla, se observa que no existe diferencia estadística entre los compuestos que contienen harina de madera y harina de corteza, a igual contenido de harina.

Otro aspecto importante observable en la tabla 3, columna 2, es que la densidad aumenta con el incremento de ambos tipos de harina. Según Beg y Pickering 2008a, esta densificación incrementa varias propiedades mecánicas de este tipo de materiales. Sin embargo, la dureza y la resistencia a la tracción de estos compuestos, sobre todo de los compuestos con altos contenidos de harina, tienden a disminuir, como se aprecia en las columnas 2, 3 y 4 . Puede que estas menores propiedades mecánicas se deban más a la pobre dispersión de las harinas en la matriz termoplástica que se produce a altos contenidos de harina, que a la densificación del compuesto (Rowell et al. 1998, Wolcott y Englund 1999, Clemons 2002, Wang et al. 2003).

\section{Eficiencia de la densidad y el contenido de harina en las propiedades físicas y mecánicas de los compuestos}

Para determinar el efecto de la densidad aparente o del contenido de harina, o de ambos, en las propiedades físicas y mecánicas de los compuestos, se siguió la metodología estadística empleada por Valenzuela y Nakayama (1991), y Valenzuela et al. (1999). Esta metodología consiste en utilizar el Análisis de Regresión Lineal Múltiple (ARLM), donde las propiedades físicas y mecánicas son tratadas como variables dependientes, y la densidad con el contenido de harina son tratadas como variables independientes. El proceso de detección se realiza en el ARLM por intermedio de un F-test de los coeficientes parciales de regresión asociados a cada variable independiente, y luego se utiliza el método paso a paso (Stepwise Method, SWM), el cual rechaza las variables independientes que no afectan a la variable dependiente, pero también, considera los efectos interactivos de las variables independientes. 
El efecto de la densidad o del contenido de harina, o de ambos, sobre las propiedades físicas y mecánicas de los compuestos determinadas por el SWM, se entregan en tabla 4, para la absorción e hinchamiento de los compuestos en base a harina de madera, en tabla 5, para la absorción e hinchamiento de los compuestos en base a harina de corteza y en tabla 6, para el módulo de elasticidad en tracción (E), la resistencia a la tracción y a la penetración de ambos tipos de compuestos.

Tabla 4. Resultados de ARLM determinados por el SWM para el \% de agua absorbida y el \% de hinchamiento de los compuestos fabricados con harina de madera.

\begin{tabular}{|c|c|c|c|c|c|c|c|}
\hline $\begin{array}{l}\text { Formulaciones- } \\
\text { Madera-PEr }\end{array}$ & Parámetro & B & $\mathrm{EE} \beta$ & $\mathbf{t}(9)$ & Valor-p & $\mathbf{R}^{2}$ & EEE \\
\hline \multirow[t]{2}{*}{$\begin{array}{c}\% \text { de agua absorbida } \\
\text { a } 25^{\circ} \mathrm{C}\end{array}$} & Intercepto & -0.0056 & 0.0042 & -1.326 & 0.2146 & 0.7834 & 0.0084 \\
\hline & Contenido de Harina & 0.8850 & 0.0001 & 6.013 & 0.0001 & & \\
\hline \multirow[t]{2}{*}{$\begin{array}{c}\text { \% de agua absorbida } \\
\text { a } 90^{\circ} \mathrm{C}\end{array}$} & Intercepto & -0.0056 & 0.0068 & 0.2008 & 0.8448 & 0.9018 & 0.0141 \\
\hline & Contenido de Harina & 0.8850 & 0.0001 & 9.5837 & 0.0000 & & \\
\hline \multirow[t]{2}{*}{$\begin{array}{l}\text { \% Hinchamientoa } \\
25^{\circ} \mathrm{C}\end{array}$} & Intercepto & 0.0012 & 0.0031 & 0.4077 & 0.6920 & 0.7432 & 0.0065 \\
\hline & Contenido de Harina & 0.8620 & 0.0000 & 5.3798 & 0.0003 & & \\
\hline \multirow[t]{2}{*}{$\begin{array}{c}\% \text { Hinchamiento } \\
\text { a } 90^{\circ} \mathrm{C}\end{array}$} & Intercepto & 0.0049 & 0.0065 & 0.7503 & 0.4703 & 0.8961 & 0.0136 \\
\hline & Contenido de Harina & 0.9466 & 0.0001 & 9.2874 & 0.0000 & & \\
\hline
\end{tabular}

En tabla 4, se entregan los resultados del ARLM determinados por el SWM, relacionando tanto la absorción como el hinchamiento con el contenido de harina, para los compuestos con harina de madera. Los resultados indican que la densidad del compuesto no fue considerada por el SWM, esto significa que el contenido de harina de madera afecta las propiedades físicas de los compuestos.

Tabla 5. Resultados de ARLM determinados por el SWM para el \% de agua absorbida y el \% de hinchamiento de los compuestos fabricados con harina de corteza.

\begin{tabular}{|c|c|c|c|c|c|c|c|}
\hline $\begin{array}{c}\text { Formulaciones } \\
\text { Corteza-PEr }\end{array}$ & Parámetro & B & $\mathrm{EE} \beta$ & $\mathbf{t}(9)$ & Valor-p & $\mathbf{R}^{2}$ & EEE \\
\hline \multirow[t]{2}{*}{$\begin{array}{c}\% \text { de agua absorbida } \\
\text { a } 25^{\circ} \mathrm{C}\end{array}$} & Intercepto & -0.00007 & 0.0010 & -0.064 & 0.949 & 0.778 & 0.002 \\
\hline & $\begin{array}{l}\text { Contenido } \\
\text { de Harina }\end{array}$ & 0.88220 & 0.0000 & 5.924 & 0.000 & & \\
\hline \multirow[t]{2}{*}{$\begin{array}{c}\% \text { de agua absorbida } \\
\text { a } 90^{\circ} \mathrm{C}\end{array}$} & Intercepto & 0.00290 & 0.0067 & 0.430 & 0.676 & 0.770 & 0.014 \\
\hline & $\begin{array}{l}\text { Contenido } \\
\text { de Harina }\end{array}$ & 0.88220 & 0.0002 & 5.802 & 0.000 & & \\
\hline \multirow[t]{2}{*}{$\begin{array}{c}\% \text { Hinchamiento } \\
\text { a } 25^{\circ} \mathrm{C}\end{array}$} & Intercepto & 0.00330 & 0.0015 & 2.234 & 0.049 & 0.379 & 0.003 \\
\hline & $\begin{array}{l}\text { Contenido } \\
\text { de Harina }\end{array}$ & 0.61618 & 0.0000 & 2.474 & 0.032 & & \\
\hline \multirow[t]{2}{*}{$\begin{array}{c}\text { \% Hinchamiento } \\
\text { a } 90^{\circ} \mathrm{C}\end{array}$} & Intercepto & 0.00596 & 0.0058 & 1.031 & 0.326 & 0.783 & 0.012 \\
\hline & $\begin{array}{l}\text { Contenido } \\
\text { de Harina }\end{array}$ & 0.88520 & 0.0001 & 6.017 & 0.000 & & \\
\hline
\end{tabular}


Los mismos resultados se encontraron para los compuestos con harina de corteza, como puede ser observado en tabla 5. Esta relación de cantidad de harina en el compuesto, con las propiedades de absorción de agua e hinchamiento, está asociada a las características higroscópicas tanto de la corteza como de la madera, sin embargo, de acuerdo a los resultados presentados en tabla 2, los compuestos fabricados con harina de corteza presentan menor absorción e hinchamiento que los compuestos fabricados con harina de madera, debido probablemente a que presentan mayor cantidad de grupos funcionales hidrofóbos de los compuestos fenólicos presentes en las paredes celulares de la corteza (Sjöström 1993, Hon y Shiraishi 2000, Rowell et al. 2005).

Cuando la resistencia a la tracción de los compuestos fue correlacionada con su densidad y su contenido de harina en ARLM, y seguido por el SWM, el contenido de harina para ambos tipos de compuestos fue elegido como la variable independiente que más afecta esta propiedad mecánica, como se muestra en tabla 6, columna 3, filas 2 y 3 . Esto significa que la densificación del compuesto no influye sobre la resistencia a la tracción de ambos tipos de compuestos. Mientras que para el módulo de elasticidad (E), el SWM consideró al contenido de harina como variable independiente que sólo afecta el E de los compuestos fabricados con harina de corteza, en cambio, para los compuestos fabricados con harina de madera, el SWM eligió a la densidad aparente del compuesto, como se puede apreciar en tabla 6, columna 4, fila 4 y 5.

Tabla 6. Resultados de ARLM determinados por el SWM para la resistencia a la tracción, módulo de elasticidad y dureza Rockwell de los compuestos fabricados con harina de madera y de corteza.

\begin{tabular}{|c|c|c|c|c|c|c|c|}
\hline $\begin{array}{c}\text { Parámetro } \\
\text { Corteza-PEr }\end{array}$ & Parámetro & B & $\mathrm{EE} \beta$ & $t(9)$ & $\begin{array}{l}\text { Valor- } \\
\text { p }\end{array}$ & $\mathbf{R}^{2}$ & EEE \\
\hline \multirow[t]{2}{*}{$\begin{array}{l}\text { Resistencia a la tracción } \\
\text { Madera-PEr(MPa) }\end{array}$} & Intercepto & 39.253 & 1.3878 & 28.284 & 0.0000 & 0.882 & 2.87 \\
\hline & $\begin{array}{c}\text { Contenido de } \\
\text { Harina }\end{array}$ & -0.9392 & 0.0370 & -8.649 & 0.0000 & & \\
\hline \multirow[t]{2}{*}{$\begin{array}{l}\text { Resistencia a la tracción } \\
\text { Corteza-PEr (MPa) }\end{array}$} & Intercepto & 43.343 & 1.6015 & 27.063 & 0.0000 & 0.903 & 3.31 \\
\hline & $\begin{array}{c}\text { Contenido de } \\
\text { Harina }\end{array}$ & -0.9507 & 0.0428 & -9.697 & 0.0000 & & \\
\hline \multirow[t]{2}{*}{$\begin{array}{l}\text { Módulo de elasticidad } \\
\text { Madera-PEr (GPa) }\end{array}$} & Intercepto & 6.097 & 0,1099 & 55,462 & 0.0000 & 0.959 & 0.23 \\
\hline & Densidad & 0.9797 & 0,0029 & 15,476 & 0.0000 & & \\
\hline \multirow[t]{2}{*}{$\begin{array}{l}\text { Módulo de elasticidad } \\
\text { Corteza-PEr (GPa) }\end{array}$} & Intercepto & 7.1087 & 0.6867 & 10.352 & 0.0001 & 0.3626 & 1.42 \\
\hline & $\begin{array}{c}\text { Contenido de } \\
\text { Harina }\end{array}$ & 0.6022 & 0.0183 & 2.3854 & 0.0385 & & \\
\hline \multirow[t]{2}{*}{$\begin{array}{c}\text { Dureza } \\
\text { Madera-PEr (Kgf) }\end{array}$} & Intercepto & 471.30 & 99.899 & 4.717 & 0.0001 & 0.367 & 22.04 \\
\hline & Densidad & -0.6063 & 99.052 & -3.234 & 0.0045 & & \\
\hline \multirow[t]{2}{*}{$\begin{array}{c}\text { Dureza } \\
\text { Corteza-PEr (Kgf) }\end{array}$} & Intercepto & 164.126 & 9.3371 & 17.577 & 0.0000 & 0.223 & 24.95 \\
\hline & $\begin{array}{c}\text { Contenido de } \\
\text { Harina }\end{array}$ & -0.4729 & 0.2495 & -2.277 & 0.0352 & & \\
\hline
\end{tabular}

En el caso de la dureza para los compuestos de harina de madera, el proceso estadístico determinó que la densidad del compuesto afecta a esta propiedad mecánica, ver tabla 6, fila 4 . En cambio, para los compuestos de harina de corteza, el procedimiento estadístico señaló al contenido de harina, 
ver tabla 6, fila 9. Sin embargo, estos modelos presentan las menores correlaciones (R2) comparados con los otros modelos obtenidos, los que fueron de 0.367 y 0.223 , respectivamente (ver tabla 6 , columna 7 , filas 7 y 9 ).

\section{CONCLUSIONES}

De acuerdo a los resultados obtenidos en esta investigación se concluye lo siguiente:

Se puede obtener compuestos en base a harina de madera y de corteza en una matriz reciclada de PEr con aceptables propiedades físicas y mecánicas.

La absorción e hinchamiento aumentó con el incremento de ambos tipos de harinas. Sin embargo, los compuestos fabricados con harina de madera absorbieron y se hincharon más que los compuestos fabricados con harina de corteza.

La resistencia a la tracción de los compuestos disminuyó con el incremento de ambos tipos de harinas, y la dureza fue menor a altos contenido de harina. Mientras que el módulo de elasticidad aumentó con el incremento de harina, y este fue mayor para los compuestos fabricados con harina de corteza que para los compuestos fabricados con harina de madera.

El compuesto con $20 \%$ de contenido de harina fue el que presentó mejores propiedades físicas y mecánicas que el resto de los compuestos, y similares o mejores propiedades físicas y mecánicas que el PEr.

El contenido de harina fue la variable que produce un mayor efecto sobre todas las propiedades de los compuestos, excepto para el módulo de elasticidad y la dureza de los compuestos fabricados con harina de madera, los que fueron más afectados por la densidad.

\section{AGRADECIMIENTOS}

Los autores agradecen la colaboración del Sr. Guillermo Soto S., candidato a Dr. en Ciencias Forestales, del programa de Postgrado en Ciencias Forestales, de la Facultad de Ciencias Forestales de la Universidad de Concepción, por la realización de los ensayos físicos y mecánicos de los compuestos, efectuados en el Laboratorio de Silvotecnología de la Madera.

\section{BIBLIOGRAFÍA}

Akil, H.; Wei Cheng, L.; Ishak, Z.A. M.; Abu Bakar, A.; Abd Rahman, M.A. 2009. Water absorption study on pultruded jute fibre reinforced unsaturated polyester composites. Composites Science and Technology 69 (11-12): 1942-1948.

Ashori, A.; Nourbakhsh, A. 2010. Reinforced polypropylene composites: Effects of chemical compositions and particle size. Bioresources Tech. 101(7): 2515-2519.

American Society for Testing and Materials, ASTM. 2008. Standard Test Methods for Density and Specific Gravity (Relative Density) of Plastics by Displacement. ASTM D 792-08. [online] $<$ http://www.astm.org/Standards/D792.htm>.

American Society for Testing and Materials, ASTM. 2010. Standard Test Method for Tensile Properties of Plastics. ASTM D 638-10. [online] <http://www.astm.org/Standards/D638.htm>. 
American Society for Testing and Materials, ASTM. 2008. Standard Test Method for Rockwell Hardness of Plastics and Electrical Insulating Materials. ASTM D 785-08. [online]

$<$ http://www.astm.org/Standards/D785.htm>.

American Society for Testing and Materials, ASTM. 2010. Standard Test Method for Water ASTM D 570-98e1. Absorption of Plastics. [online] <http://www.astm.org/Standards/D570.htm>.

Beall, F.C.; Eickner, H.W. 1970. Thermal Degradation of Wood Components, a Review of the Literature. Forest Products Laboratory, Madison, Wisconsin, USA, FPL 130: 1-27.

Beg, M.D.H.; Pickering, K.L. 2008a. Part I: Effects on physical and mechanical properties. Composites Part A: Applied Science and Manufacturing 39(7): 1091-1100.

Beg, M.D.H.; Pickering, K.L. 2008b. Reprocessing of wood fibre reinforced polypropylene composites Part II: Hygrothermal ageing and its effects. Composites Part A: Applied Science and Manufacturing 39(7): 1565-1571.

Berrocal, A.; Freer, J.; Baeza, J.; Rodríguez, J. y Espinosa, B., 2004. Relación entre edad del árbol y su composición química en Pinus radiata (D. Don) crecido en Chile y su importancia para la producción de bioetanol. Kurú: Revista Forestal 1(1):1-8. Costa Rica.

Bledzki, A. K.; Faruk, O. 2004. Creep and impact properties of wood fibre-polypropylene composites: influence of temperature and moisture content. Comp. Sc. and Tech. 64(5): 693-700.

Bouafif, H.; Koubaa, A.; Perré, P.; Cloutier, A. 2009-a. Effects of fiber characteristics on the physical and mechanical properties of wood plastic composites. Composites Part A: Applied Science and Manufacturing 40(12): 1975-1981.

Bouafif, H.; Koubaa, A.; Perré, P.; Cloutier, A.; Riedl, B. 2009-b. Wood Particle/HDPE Composites: Thermal Sensitivity and Nucleating Ability of Wood Particles. Journal of App. Pol. Sc.113(1): 593-600.

Bozaci, A.; Öktem, T.; Seventekin, N. 2007. Pineapple fiber. Tektil ve konfeksiyon 3:163-169.

Clemons, C. 2002. Wood-plastics composites in the United States. Forest Products Journal 52 (6): $10-18$.

Demir, H.; Atikler, U.; Balköse, D.; Tihminlioglu, F. 2006. The effect of fiber surface treatments on the tensile and water sorption properties of polypropylene-luffa fiber composites. Composites Part A: Applied Science and Manufacturing 37(3): 447-456.

Dominkovics, Z.; Dányádi, L.; Pukánszky, B. 2007. Surface modification of wood flour and its effect on the properties of PP/wood composites. Composites Part A: Applied Science and Manufacturing 38(8): 1893-1901.

English, B.; Stark, N.; Clemons, C. 1997. Weight Reduction: Wood versus Mineral Fillers in Polypropylene Fourth International Conference on Wood fiber-Plastic Composites. May 12-14, Madison, Wisconsin, USA: 237-246.

English, B. 1999. 40\% Wood Flour-PP. Proceeding $4^{\text {th }}$ International Conference on Wood Fiber Plastic Composites. Madison. USA, pp:134-142. 
Gardner, D.; Han, Y.; Song, W. 2008. Wood Plastic Composites Technology Trends in Proceedings of the 51st International Convention of Society of Wood Science and Technology. November 10-12, 2008 Concepción, CHILE. Paper WS-24: 1-6.

Godara, A.; Raabe, D.; Bergmann, I.; Putz, R.; Müller, U. 2009. Influence of additives on the global mechanical behavior and the microscopic strain localization in wood reinforced polypropylene composites during tensile deformation investigated using digital image correlation. Composites Science and Technology 69(2): 139-146.

Guo, G.; Rizvi, G. M.; Park, C. B.; Lin, W. S. 2004. Critical Processing Temperature in the Manufacture of Fine-Celled Plastic/Wood-Fiber Composite Foams. J. of App. Pol. Sc. 91(1): 621-629.

Hakki, A. M.; Kelley, S. 2002. The application of pyrolysis-molecular beam mass spectrometry for characterization of barkphenolysis products. Biomass and Bioenergy 22(5): 411- 419.

Harper, D.; Wolcott, M. 2004. Interaction between coupling agent and lubricants in wood-PP composites. Composites Part A: Applied Science and Manufacturing 35(3): 385-394.

Hon, D.; Shiraishi, N. 2000. Wood and Cellulosic Chemistry. 2da Edition. CRC Press 8 Nov.: 914.

Instituto Nacional de Normalizacion, INN. 1980. Paneles a base de madera, determinación de las dimensiones y formas. NCh No 761. Santiago, Chile. [on line] <http://www.inn.cl/busquedas/ busqueda/detalle_de_busqueda.asp?cd=NCh761.Of1980> [Consultado: 10 de abril 20011].

Jayaraman, K.; Bhattacharyya, D. 2004. Mechanical performance of wood fiber-waste plastic composite materials. Resources Construction and Reycling 41(4): 307-319.

Kandem, D.; Jiang, H.; Cui, W.; Freed, J.; Matuana, L. 2004. Properties of wood plastic composites made of recycled HDPE and wood flour from CC-treated wood removed from service. Composites: Part A 35: 347-355.

Kim, S.; Moon, J.; Kim, G.; Ha, Ch. 2008. Mechanical Properties of PP/natural fiber composites: Comparison of wood fiber and cotton fiber. Polymer Testing 27(7): 801-806.

Kuan, Ch.; Kuan, H.; Ma, Ch.; Chien-Ming, H., 2006. Mechanical, thermal and morphological properties of water-cross linked wood flour reinforced linear low-density polyethylene composites. Composites Part A: Applied Science and Manufacturing 37(10): 1696-1707.

Kuo, P.; Wang, S.; Chen, J.; Hsueh, H.; Tsai, M. 2009. Effects of material compositions on the mechanical properties of wood-plastic composites manufactured by injection molding. Materials and Design 30(9): 3489-3496.

Lee, SY.; Kang, IA.; Doh, GH.; Yoon, HG.; Park, BD.; Wu, Q. 2008. Thermal and mechanical properties of wood flour/talc-filled polylactic acid composites: effect of filler content and coupling treatment. J. Thermoplastic Composite Materials 21(3): 209-230.

Lucchetta, G.;Bariani, P.F.; Knight, W.A. 2006. A New Approach to the Optimization of Blends Composition in Injection Moulding of Recycled Polymers. Annals of the CIRP 55(1): 1-4.

Matuana, L.; Park, Ch.; Balatinecz, J. 1997. Processing and Cell Morphology Relationships for Microcellular Foamed PVC/Wood-Fiber Composites. Polymer Eng. and Sc. 37(7): 1137-1147. 
Meran, C.; Ozturk, O.; Yuksel, M. 2008. Examination of the possibility of recycling and utilizing recycled polyethylene and polypropylene. Materials and Design 29: 701-705.

Najafi, S.; Kiaefar, A.; Tajvidi, M. 2008. Effect of Bark Flour Content on the Hygroscopic Characteristics of Wood-Polypropylene Composites. Journal of App. Pol. Sc. 110(5): 3116-3120.

Neagu, R. C.; Gamstedt, E. K.; Lindström, M. 2005. Influence of wood-fibre hygroexpansion on the dimensional instability of fibre mats and composites. Composites Part A: Applied Science and Manufacturing 36(6): 772-788.

Nemli, G.; Kirc, H.; Temiz, A. 2004. Influence of impregnating wood particles with mimosa bark extract on some properties of particleboard. Industrial Crops and Products 20(3):339-344.

Ngueho, M.; Koubaa, A.; Cloutier, A.; Soulounganga, P.; Wolcott, M. 2010. Effect of bark fiber content and size on the mechanical properties of bark/HDPE composites. Composites Part A: Applied Science and Manufacturing 41: 131-137.

Panthapulakkal, S.; Sain, M. 2007. Agro-residue reinforced HDPE composites: Fiber characterization and analysis of composite properties. Composites Part A: Applied Science and Manufacturing 38(6): 1445-1454.

Poblete, H.; Sánchez, J. 1991. Tableros con corteza de P. radiata D. Don. Bosque 12(1): 17-26.

Rahman, R.; Huque, M.; Islam, N.; Hasan, M. 2009. Mechanical properties of polypropylene composites reinforced with chemically treated abaca. Composites Part A: Applied Science and Manufacturing 40(4): 511-517.

Raukola, J.; Makinen, K, 2003. Wood plastic composites with conical Conex Wood Extruder, Wood Fiber-Composites, report 31.12.2003, VTT. Finland.pp 1-7.

Renner, K.; Móczó, J.; Pukanszky, B. 2009. Deformation and failure of PP composites reinforced with lignocellulosics fibers: Effect of inherent strength of the particles. Composites Science and Technology 69(10): 1653-1659.

Rizvi, G.; Matuana, L.; Park, C. 2000. Foaming of PS/Wood Fiber Composites Using Moisture as a Blowing Agent. Polymer Engineering and Science 40(10): 2124-2132.

Rowell, R. M.; Caulfield, D. F.; Chen, G.; Dale, Ellis W.; Jacobson, R. E.; Lange, S.; Schumann, R. 1998. Recent advances in agro fiber thermoplastic composites. 2th Int. Symp. on Natural Polymers and Composites - ISNaPol/98. Atibaia, SP, Brasil. 22 pp.

Rowell, R.; Lange, S.; Jacobson, R. 2000. Weathering Performance of Plant-Fiber/ Thermoplastic Composites. Molecular Crystals and Liquid Crystals 353(1): 85-94.

Rowell, R.; Pettersen, R.; Han, J.; Rowell, J. 2005. Cell Wall Chemistry in Handbook of Wood Chemistry and Wood Composites. CRC Press LLC, Boca Raton, Florida, USA: 35-76.

Sanadi, A. R.; Caulfield, D. F.; Jacobson, R. E.; Rowell, R. M. 1995. Renewable Agricultural Fibers as Reinforcing Fillers in Plastics: Mechanical Properties of Kenaf Fiber-Polypropylene Composites. Industrial Engineering Chemistry Research 34(5): 1889-1896. 
Sanadi, A.; Caulfield, D.; Stark, N.; Clemons, C. 1999. Thermal and mechanical analysis of lignocellulosic polypropylene composites. The Fifth International Conference on Wood fiber-Plastic Composites. May 26-27, Mad., USA: 67-79.

Selke, S.; Wichman, I. 2004. Wood fiber/polyolefin composites. Composites Part A: Applied Science and Manufacturing 35(3): 321-326.

Shebani, A.N.; Van Reenen, A.J.; Meincken, M. 2009. The effect of wood extractives on the thermal stability of different wood-LLDPE composites. Thermochimica Acta 481(1-2): 52-56.

Sjöström, E. 1993. Wood Chemistry. Cap I y II, Academic Press, London, England.pp 17-378.

Stark, N. 1997. Effect of Species and Particle Size on Properties of Wood-Flour-Filled Polypropylene Composites. Proceedings of Functional Fillers December 8-10, Le Meridien at Coronado, San Diego, California: 1-21.

Stark, N. 1999. Wood fiber derived from scrap pallets used in polypropylene composites. Forest Products Journal 49(6): 39-46.

Valenzuela, L.; Nakayama, Y. 1991. Predicting MOR of radiata pine grown in Chile, efficiency of some nondestructive parameters. Mokusai Kogyo 46 (5): 213-219.

Valenzuela, L.; Miyoshi, K.; Moya, R. 1999. Eficiencia de algunos parámetros afectando al MOE en flexión del Pinus radiata. Maderas: Ciencia y Tecnología 1(2): 37-46.

Wambua, P.; Ivens, J.; Verpoest, I. 2003. Natural fibres: can they replace glass in fibre reinforced plastics?. Composites Science and Technology 63(9): 1259-1264.

Wang, B.; Panigrahi, S.; Tabil, L.; Crerar, W.; Powell, T.; Kolybaba, M.; Sokhansanj, S., 2003. Flax Fiber-Reinforced Thermoplastic Composites, en Annual Intersectional Meeting Red River of ASAE Quality Inn \& Suites, 301 3rd Avenue North Fargo, North Dakota, USA, October 3-4: 1-14.

Wang, W.; Morrell, J. J. 2004. Water sorption characteristics of two wood-plastic composites. Forest Products Journal 54(12): 209-214.

Wolcott, M.; Englund, K. 1999. A Technology Review of Wood Plastic Composites. In Proceeding 33th Int. Particleboard Composites Material Symposium, Abril 13-15: 1-14. Madison, USA.

Woodhams, RT, Thomas, G, Rodgers DK, 1984. Woodfibres as reinforcing fillers for polyolefins. Polymer Engeneer and Science. 24(15):1166-1171.

Yadama, V.; Lowell, E.; Peterson, N.; Nicholls D. 2009. Wood-Thermoplastic Composites Manufactured Using Beetle-Killed Spruce from Alaska. Polymer Eng. and Sc. 49 (1):129-136.

Yang, H.; Kim, H.; Park, H.; Lee, B.; Hwang, T. 2006-a. Water absorption behavior and mechanical properties of lignocellulosic filler-polyolefin bio-composites. Composite Structures 72(4): 429-437.

Yang, H.; Wolcott, M. P.; Kim, H.; Kim, S.; Kim, H. 2006-b. Properties of lignocellulosic material 
filled PP bio-composites made with different manufacturing processes. Pol. Testing. 25: 668-676.

Yeh, Sh.; Agarwal, S.; Gupta, R. K. 2009. Wood-plastic composites formulated with virgin and recycled ABS. Composites Sc. and Tech. 69(13): 2225-2230.

Yin, S.; Rials, T.; Wolcott, M. 2001. Crystallization behavior of polypropylene and its effect on wood fiber composite properties. Proceeding Sixth International Conference on Wood fiber-Plastic Composites. Forest Product Society: 139-146.

Youngquist, J.; Myers, G. E.; Muehl, J. H.; Krzysik, A. M.; Clemons, C. M. 1995. Composites from Recycled Wood and Plastics. EPA Project Summary, Engineering Laboratory Cincinnati, USA.

Zabihzadeh, M. 2010. Water uptake and flexural properties of natural filler/HDPE composites. Bioresources 5(1): 316-323.

Zadorecki, P.; Mitchell, AJ. 1989. Future prospects for wood cellulose as reinforcement in organic polymer composites. Polymer Composites 10 (2):69-77. 
